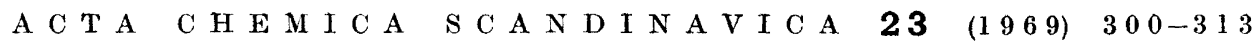

\title{
On the Crystal Structure of the Trigonal (and Cubic) Phase of Potassium Nitrite
}

\author{
J. K. S OL B A K K and K. O. S T R $\emptyset$ M M E \\ Department of Chemistry, University of Oslo, Blindern, Oslo 3, Norway
}

The crystal structure of potassium nitrite has been determined from three-dimensional X-ray data obtained at room temperature. The trigonal unit cell of dimensions $a=b=5.011(0.0012) \AA$ and $c=10.153(0.0054) \AA$ contains three units of $\mathrm{KNO}_{2}$. The statistical space group symmetry is $R \overline{3} m$ in accordance with recent reports. ${ }^{1,2}$

When averaged in time, the nitrite group acquires a threefold statistical symmetry with the nitrogen atoms lying in the mirror planes of the space group, while the oxygen atoms are slightly displaced from these planes. The nitrogen atom acquires a formal positive charge. The structure resembles closely that of phase I potassium nitrate, which is stable above $128^{\circ} \mathrm{C}$. This similarity provides an explanation of the observed partial solubility of potassium nitrate in solid potassium nitrite. ${ }^{3}$ The solubility has been determined to be $15 \pm 2 \%$ at room temperature. It has also been confirmed that commercially available potassium nitrite contains several per cent of potassium nitrate. ${ }^{4}$ The phase diagram of the ternary system $\mathrm{KNO}_{2}$, $\mathrm{KNO}_{3}, \mathrm{H}_{2} \mathrm{O}$ is discussed. ${ }^{3}$

The configurational entropy, which was calculated to be $\mathbf{3 . 5 6}$ $\mathrm{cal} / \mathrm{mole} \mathrm{deg}$, is discussed in relation to the change in entropy observed at the transformation between the low temperature and room temperature phases. ${ }^{5}$

On the basis of $\mathrm{X}$-ray diffraction data obtained for crystals grown from aqueous solution at $-20^{\circ} \mathrm{C}$, the low temperature phase of potassium nitrite has been found to be monoclinic, and unit cell dimensions are given. A structural model of the cubic phase, which is stable above $40^{\circ} \mathrm{C}, 4,6$ is also discussed.

$\mathrm{T}$ he present work was undertaken as part of a study of "disordered" solids since the anomalously high heat of transition at the first order transformation occurring at $-13^{\circ} \mathrm{C}, 5$ and the soft nature of the crystals at room temperature, suggest the room temperature phase to be disordered. Ziegler's report that the crystals are monoclinic, ${ }^{7}$ was followed during the course of the present work by two independent notes classifying the structure as rhombohedral.1,2 These deductions are in accordance with our own conclusions. Both authors 
reported the structure to be disordered, and Chang ${ }^{1}$ proposed a structural model on the basis of space group $R \overline{3} m$.

According to Ray, ${ }^{4}$ the room temperature phase transforms by means of a first order process at $39.1^{\circ} \mathrm{C}$ into a high temperature form. Ubbelohde et al ${ }^{6}$ report this transition to be continuous, and the high temperature phase to be cubic with a unit cell axis of $6.66 \AA$ at $45^{\circ} \mathrm{C} .^{8}$

\section{EXPERIMENTAL}

Short, almost spherical crystals of potassium nitrite were prepared by slow evaporation of water from a nearly saturated aqueous solution of reagent grade material from Riedel de Haen AG in a desiccator at room temperature. Single crystals of approximate dimensions $0.15 \times 0.15 \times 0.15 \mathrm{~mm}^{3}$ were rapidly transferred from the solution to thin walled boron-lithium capillaries which were then sealed off. Two sets of integrated equiinclination Weissenberg photographs of the reflections of the first four layers were ob. tained, with rotation of the specimens through $200^{\circ}$ about the [100] and [010] directions referred to monoclinic indexing, respectively, using filtered $\mathrm{Cu} K \alpha$ radiation. Two different crystals were used in the measurements, one for each set. All of the measurable spot intensities of the five photographs for each layer were obtained photometrically, while the weakest intensities were estimated visually. The mean intensity data for each layer (normalized to a common scale) were corrected for the Lorentz and polarization effects in the usual way. The two sets of data were then normalized to a common scale and average values of the intensities obtained in accordance with the monoclinic symmetry conditions. The number of independent reflections having non-zero intensities is 125 (corresponding to $\sin \theta<0.85$ ), which reduces to 60 independent reflections in rhombohedral indexing.

The unit cell dimensions of potassium nitrite containing $3 \%$ by weight of potassium nitrate (reagent grade); of pure potassium nitrite; and of potassium nitrate grown from an aqueous solution which was almost saturated with potassium nitrite; were refined by least squares techniques in which 5, 4, and 15 resolved and indexed lines were used, respectively. These were measured on powder photographs obtained with a Guinier camera at room temperature using $\mathrm{KCl}$ as a standard. In order to reduce oxidation of $\mathrm{KNO}_{2}$ to $\mathrm{KNO}_{3}$ (which has a different crystal structure at room temperature), exposure times of less than $5 \mathrm{~h}$ were used.

Oscillation and Weissenberg photographs were taken of (virtually) single crystals of the low temperature form of potassium nitrite, which were rotated about the $c$ axis. Suitably pure crystals were grown from an aqueous solution of potassium nitrite which had been prepared from silver nitrite following a method previously described. ${ }^{9}$

Crystals of potassium nitrite saturated with potassium nitrate grew from a concentrated aqueous solution in contact with solid potassium nitrate over a period of one month. The resulting crystals were distinguished from those of potassium nitrate by differences in appearance and hardness. The amount of potassium nitrate in the saturated crystals was determined according to a method described by Kolthoff. ${ }^{9}$ The composition of the commercial product was determined by titration against standard permanganate. The results are as follows: $2.8 \pm 0.3 \% \mathrm{KNO}_{3}$ in $\mathrm{KNO}_{2}$ (reagent grade) $15 \pm 2 \% \mathrm{KNO}_{3}$ in $\mathrm{KNO}_{2}$ (saturated with $\mathrm{KNO}_{3}$ ). The relatively large degree of uncertainty in the latter result is probably associated with the method of preparation.

\section{SPACE GROUP AND UNIT CELL DIMENSIONS}

At room temperature and atmospheric pressure, the crystals are rhombohedral, with the observed unit cell dimensions given in Table 1, which includes values recently reported in the literature. ${ }^{1,2}$ The present results are seen to compare well with the reported values. There are three molecules of $\mathrm{KNO}_{2}$ 
Table 1. Observed unit cell dimensions at room temperature E.s.d. in parentheses.

\begin{tabular}{|c|c|c|c|}
\hline$a(\AA)$ & $c(\AA)$ & Sample form & Sample purity \\
\hline $\begin{array}{c}5.003 \\
(0.012)\end{array}$ & $\begin{array}{c}10.228^{1} \\
(0.014)\end{array}$ & single cryst. & \\
\hline 5.043 & $10.109^{2}$ & single cryst. & \\
\hline $\begin{array}{c}5.011 \\
(0.0012)\end{array}$ & $\begin{array}{c}10.153 \\
(0.0054)\end{array}$ & $\begin{array}{l}\text { powder } \\
\text { (Guinier) }\end{array}$ & $\begin{array}{l}\text { commercial } \\
\text { (r.g.) }\end{array}$ \\
\hline $\begin{array}{c}5.006 \\
(0.0004)\end{array}$ & $\begin{array}{c}10.190 \\
(0.0021)\end{array}$ & $\begin{array}{l}\text { powder } \\
\text { (Guinier) }\end{array}$ & pure \\
\hline
\end{tabular}

per unit cell, the density of pure material is $1.917 \mathrm{~g} / \mathrm{cm}^{3}$. No super-structure reflections could be observed.

Of the centrosymmetric $R \overline{3} m$ and non-centrosymmetric $R 3 m$ space groups, the former was chosen, as the structure is clearly statistical, and the low temperature phase is apparently centrosymmetric. At $-20^{\circ} \mathrm{C}$ the crystals are monoclinic with unit cell dimensions of $a \sin \beta=4.16 \pm 0.02 \AA$, $b=9.62 \pm 0.05 \AA, c=6.19 \pm 0.03 \AA$, and $\beta=113 \pm 1^{\circ}$. The corresponding density is $1.96 \mathrm{~g} / \mathrm{cm}^{3}$. The unit cell contains four molecules, the space group symmetry being $P 2_{1} / c$. Although these crystals were grown from an aqueous solution, the phase is probably the same as the, yet unidentified, low temperature phase for which the transition points $-13^{\circ} \mathrm{C}^{5}$ and $-17.7^{\circ} \mathrm{C}^{1}$ have been reported. The former is observed in pure potassium nitrite and the latter in a sample which possibly contained potassium nitrate, as has been reported for commercially available potassium nitrite of reagent grade. ${ }^{4}$ The low temperature form of $\mathrm{KNO}_{2}$ is probably not disordered, and it is unlikely that $\mathrm{KNO}_{3}$ should show appreciable solubility in this phase. There are reasons (see later) for believing that the solid solution of the room temperature phase behaves as an ideally diluted solution. It may be shown on this basis that a depression of freezing point of $4.7^{\circ} \mathrm{C}$ corresponds to $4.7 \%$ by weight of potassium nitrate in the room temperature phase of potassium nitrite.

\section{DETERMINATION OF THE STRUCTURE}

The three potassium ions per unit hexagonal cell were placed in threefold positions, i.e. in centres of symmetry with $\mathrm{K}^{+}$in $\left(0,0, \frac{1}{2}\right)$, etc. A three dimensional Fourier analysis was based on this positioning. In addition to showing the positions of the potassium ions, the resulting electron density map revealed a maximum at the origin from which relatively high electron densities extend in the three equivalent mirror planes passing through the $c$ axis and points having the coordinates $(x, 2 x, z),(2 x, x, z)$ and $(x, \bar{x}, z)$. Fig. 1 


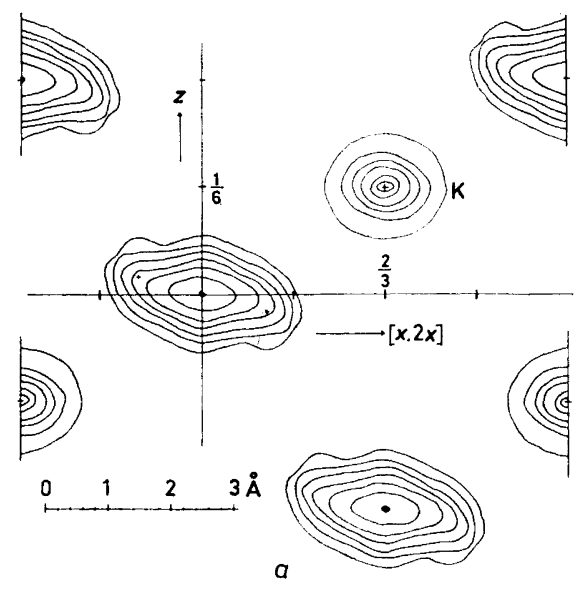

Fig. 1. a. Electron density in the mirror plane perpendicular to the $x$-axis. Contours at intervals of $0.5 \mathrm{el} . / \AA^{3}$ for the light atoms and at intervals of $0.5 \mathrm{el} . / \AA^{3}$ for potassium. The outer contour represents 1 el. $/ \AA^{3}$. b. Electron density in the plane perpendicular to the $z$-axis at $z=-0.03$. Contours at intervals of $0.5 \mathrm{el} . / \AA^{3}$. The outer contour represents 1 el. $/ \AA^{3}$. c. The contours of 2 el. $/ \AA^{3}$ in planes parallel with the $x z$-plane at $y=0,0.08,0.16$, and 0.24 .

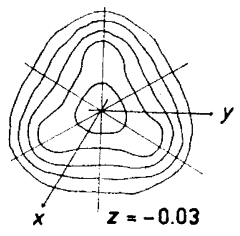

$b$

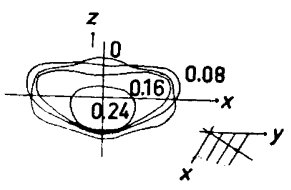

C

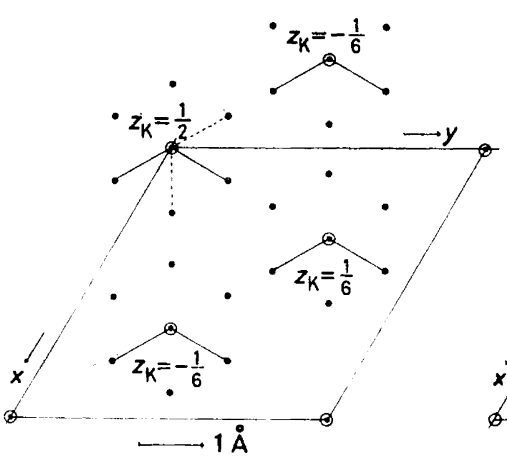

$a$

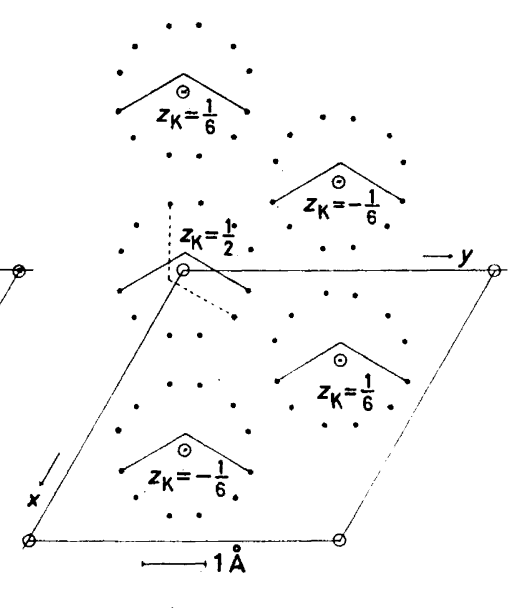

$b$

Fig. 2. a. Model of the statistical structure of potassium nitrite obtained from the electron density map. For neighbouring atoms on a threefold axis: $z_{\mathrm{K}}-z_{\mathrm{N}}= \pm \frac{1}{2}$. b. Final model of the statistical structure at room temperature. For neighbouring atoms alternately on and close to a threefold axis: $z_{\mathrm{K}}-z_{\mathrm{N}} \approx \pm \frac{1}{2}$. One of the many possible molecular configurations is indicated.

Acta Chem. Scand. 23 (1969) No. 1 
shows the electron density in various regions. It seemed reasonable to place the nitrogen atom at $(0,0,0)$, etc. and oxygen statistically in the mirror planes, i.e. in the special positions termed $18 \mathrm{hm}$ in the International Tables. This corresponds to the statistical model of Chang, ${ }^{1}$ which is shown in Fig. 2a. The positions of the oxygen atoms were calculated on the basis of an $\mathrm{N}-\mathrm{O}$ bond distance of $1.24 \AA$ and ONO bond angle of $115^{\circ}$ as has been reported for sodium nitrite, ${ }^{10}$ since the oxygen atoms did not give rise to separate peaks on the map. The two oxygen atoms of the nitrite group occupy positions such as $(x, 2 x, z)$ and $(x, \bar{x}, z)$, respectively. The positions thus obtained for the oxygen atoms are within the regions of relatively high electron density extending from the origin as mentioned above.

The introduction of the contributions to the structure factors from all atoms using a common isotropic temperature factor of $7 \AA^{2}$ did not, however, lead to any alterations in the signs of the structure factors. The lack of resolution of the oxygen peaks arises from three-dimensional over-lap of the electron density of nitrogen and oxygen atoms, and it is questionable whether the nitrogen atom is not also positionally disordered.

The structure was then refined using a full matrix L.S. programme. The positions of the atoms were as follows: $\mathrm{K}:\left(0,0, \frac{1}{2}\right)$, etc., $\mathrm{N}:(0,0,0)$, etc., $\mathrm{O}$ : $(x, 2 x, z)$, etc., where the input parameters of the oxygen atoms were as previously obtained. Values of the anisotropic temperature factor coefficients were computed from the expression

$$
\exp -\left(\beta_{11} h^{2}+\beta_{22} k^{2}+\beta_{33} l^{2}+\beta_{12} h k+\beta_{13} h l+\beta_{23} k l\right)
$$

Non-observed reflections were excluded in the L.S. calculations. The weights $w$, attached to the reflections were computed from the formula $w=\left(a+F+c \cdot F^{2}\right)^{-\frac{1}{2}}$, where $a=2 F(\min )$ and $c=2 / F(\max )$. The values of the form factors for the neutral atoms which were used in the structure factor calculations, ${ }^{11}$ were multiplied by constants corresponding to excess charges of the following, somewhat arbitrary, values (in electron units) associated with the various atoms: $n(\mathrm{~K})=0.8, n(\mathrm{~N})=0.4$ and $n(\mathrm{O})=-0.6$. A positive excess charge on the nitrogen atom is indicated by the large $\mathrm{N}-\mathrm{K}$ distance. A similar charge distribution has been obtained from an analysis of X-ray diffraction data for potassium nitrate (to be published). See also Refs. 12 and 13.

As a result of the L.S. refinement, the $R$-factor fell to $5.67 \%$. The $\mathrm{N}-\mathrm{O}$ bond distance thus obtained is $1.054 \AA$ compared with a normal bond length of $1.25 \AA^{10}$ The angle between the bond directions is found to be $3^{\circ}$ less than the expected value of $115^{\circ} .^{10}$ It appeared that the thermal motion is generally very strong. The apparent motion of the oxygen atom is especially vigorous in the direction perpendicular to the mirror plane. An estimate of the correction of the $\mathrm{N}-\mathrm{O}$ bond length due to librational motion was made following the methods outlined by Cruickshank. ${ }^{14}$ The translational motion was assumed to be isotropic and the librational motion about the orthogonal $x$ axis equal to that about the $y$ axis. The correction, which amounted to $0.025 \AA$, is probably correct only to the order of magnitude.

The standard deviation in the $\mathrm{N}-\mathrm{O}$ bond length derived on the basis of the estimated uncertainty in the values of the $x$ and $y$ parameters of oxygen, is $0.023 \AA$, ignoring correlation effects. Since such effects are relatively impor- 
tant in the present case ( $c f$. , for example, Ref. 15), the quoted value is too small, although being of the correct order of magnitude.

Thus the corrections considered here are insufficient to cause the large discrepancy between the observed and normal values of the $\mathrm{N}-\mathrm{O}$ bond lengths. In order to obtain satisfactory dimensions of the nitrite group it seemed therefore necessary to place the nitrogen atoms statistically outside the origin (in the mirror planes). Furthermore, on the basis of an $\mathrm{O}-\mathrm{N}$ bond length of $1.24 \AA$ and an ONO bond angle of $115^{\circ}$, a $\mathrm{K}-\mathrm{O}$ distance of $2.59 \AA$ is obtained when the nitrogen atom is placed at the origin, and the oxygen atom in the mirror plane. This figure appears to be rather small when it is compared, for example, with the two shortest $\mathrm{K}-\mathrm{O}$ distances observed in the disordered phase of potassium nitrate, which are 2.70 and $2.79 \AA$. However, on the basis of the present position of the oxygen atom in the mirror plane, a normal $\mathrm{N}-\mathrm{O}$ bond length corresponds to a bond angle of $93^{\circ}$. Comparison with a normal bond angle of $115^{\circ}$ demonstrates in addition the need for placing the oxygen atoms outside the mirror planes.

On the other hand, Chang ${ }^{1}$ proposed the structural model obtained above (Fig. 2a). By direct minimizing of the reliability index, which leads to $R=11.2 \%$, he obtained an apparent $\mathrm{N}-\mathrm{O}$ bond length of $1.140 \AA$, corresponding to an $\mathrm{O}-\mathrm{N}-\mathrm{O}$ bond angle of $108.1^{\circ}$. After correcting for rotational effects, he obtained a bond length of $1.230 \AA$, while the bond angle could not be refined.

The $x$ and $y$ parameters of the oxygen atom were then estimated on the basis of a calculated intermolecular $\mathrm{O}-\mathrm{O}$ distance of $2.09 \AA$ and a $\mathrm{K}-\mathrm{O}$ distance of $2.7 \AA$ (between $K(1 / 3,2 / 3,1 / 6)$ and the nearest oxygen atom of the nitrite group associated with the origin in Fig. 2), and using the same $z$ parameter as was obtained previously. The $x(=2 y$ by symmetry) and the $z$ parameter of the corresponding nitrogen atom were calculated on the basis of the $\mathrm{N}-\mathrm{O}$ bond length of $1.24 \AA$, so that the distance from $\mathrm{N}$ to $\mathrm{K}$ $(-1 / 3,1 / 3,-1 / 6)$ became approximately equal to that from $N$ to $K$ $(-2 / 3,-1 / 3,1 / 6)$. These values were utilized in the subsequent L.S. calculation wherein the $x$ parameter of the oxygen atom and the $z$ parameter of the nitrogen atom were of necessity maintained constant. The thermal parameters obtained earlier were used, except for nitrogen, which was treated isotropically using a value of $15 \AA^{2}$, which is the average of the anisotropic thermal parameters obtained previously. As a result of the L.S. refinement, the $R$-factor fell to $5.4 \%$. The values of the free position parameters changed very little. Thus an $\mathrm{N}-\mathrm{O}$ bond length of $1.22 \AA$ and an ONO bond angle of $115^{\circ}$ were obtained. The thermal parameter values of the potassium ion were practically equal to those obtained previously. The anisotropy of the oxygen atom across the mirror plane was virtually eliminated, whereas the value of the thermal parameter of the nitrogen atom decreased (by $5 \AA^{2}$ ), as is to be expected if the atom is outside rather than at the origin.

The molecular parameters of the nitrite group were next put equal to the values obtained above, and kept fixed in the following L.S. calculation, whereas the nitrite group shown close to the origin in Fig. 2, was displaced in the [210] and [001] directions. The only free position parameters were the $y$ and $z$ values of the oxygen atom, the other position parameters being reset after each cycle.

Acta Chem. Scand. 23 (1969) No. 1 
Table 2. Observed and calculated structure factors.

\begin{tabular}{|c|c|c|c|c|}
\hline$H$ & $K$ & $L$ & $K F O B$ & $F \mathrm{CAL}$ \\
\hline 0 & 1 & -1 & 10.34 & 9.64 \\
\hline 0 & 2 & -2 & 25.53 & 25.11 \\
\hline 0 & $\mathbf{3}$ & -3 & 8.86 & 8.73 \\
\hline 0 & 4 & -4 & 3.10 & 3.81 \\
\hline 2 & -1 & 0 & 34.93 & 33.46 \\
\hline 2 & 0 & -1 & 24.51 & 26.41 \\
\hline 2 & 1 & -2 & 11.68 & 12.06 \\
\hline 2 & 2 & -3 & 6.65 & 6.09 \\
\hline 2 & 3 & -4 & 2.43 & 2.74 \\
\hline 4 & -2 & 0 & 8.75 & 8.54 \\
\hline 4 & -1 & -1 & 7.30 & 6.44 \\
\hline 4 & 0 & -2 & 4.95 & 4.61 \\
\hline 1 & -4 & 5 & 3.81 & 3.87 \\
\hline 1 & -3 & 4 & 11.76 & 11.85 \\
\hline 1 & -2 & 3 & 20.35 & 20.69 \\
\hline 1 & -1 & 2 & 58.72 & 62.66 \\
\hline 1 & 2 & -1 & 16.61 & 16.15 \\
\hline 1 & 3 & -2 & 6.95 & 7.04 \\
\hline 1 & 4 & -3 & 1.82 & 2.16 \\
\hline 3 & -5 & 5 & 1.80 & 1.94 \\
\hline 3 & -4 & 4 & 4.95 & 5.06 \\
\hline 3 & -3 & 3 & 12.20 & 11.83 \\
\hline 3 & 0 & 0 & 11.05 & 10.78 \\
\hline 3 & 2 & -2 & 3.96 & 3.56 \\
\hline 0 & $-\overline{4}$ & 7 & 1.98 & 1.95 \\
\hline 0 & -3 & 6 & 6.98 & 6.92 \\
\hline 0 & -2 & 5 & 10.95 & 10.67 \\
\hline 0 & $-\overline{1}$ & 4 & 41.58 & 41.91 \\
\hline 0 & 0 & 3 & 2.80 & 2.57 \\
\hline 0 & 4 & -1 & 4.19 & 4.33 \\
\hline 2 & -4 & 6 & 4.46 & 4.23 \\
\hline 2 & -3 & 5 & 11.72 & 10.60 \\
\hline 2 & -2 & 4 & 13.90 & 13.68 \\
\hline 2 & $\overline{3}$ & -1 & 2.20 & 2.77 \\
\hline 1 & -3 & 7 & 5.64 & 5.11 \\
\hline 1 & -2 & 6 & 16.11 & 13.64 \\
\hline 1 & $-\overline{1}$ & 5 & 14.25 & 14.19 \\
\hline 1 & 4 & 0 & 2.42 & 2.84 \\
\hline 3 & $-\overline{3}$ & 6 & 4.34 & 4.61 \\
\hline 0 & -3 & 9 & 2.10 & 2.23 \\
\hline 0 & -2 & 8 & 8.17 & 7.78 \\
\hline 0 & $-\overline{1}$ & 7 & 10.00 & 9.45 \\
\hline 0 & 0 & 6 & 26.22 & 28.76 \\
\hline 2 & -4 & 9 & 1.82 & 1.86 \\
\hline 2 & $-\overline{\mathbf{3}}$ & 8 & 4.36 & 4.12 \\
\hline 2 & -2 & 7 & 10.19 & 9.80 \\
\hline 1 & $-\overline{3}$ & 10 & 2.56 & 2.69 \\
\hline 1 & -2 & 9 & 5.19 & 5.06 \\
\hline 1 & $-\overline{1}$ & 8 & 11.24 & 10.24 \\
\hline 3 & -3 & 9 & 2.92 & 2.97 \\
\hline 0 & -2 & 11 & 1.85 & 2.12 \\
\hline 0 & -1 & 10 & 5.50 & 5.18 \\
\hline 0 & 0 & 9 & 6.54 & 6.93 \\
\hline 0 & 4 & 5 & 2.48 & 3.01 \\
\hline 2 & -3 & 11 & 1.64 & 1.76 \\
\hline 2 & -2 & 10 & 2.98 & 3.20 \\
\hline 1 & $-\overline{1}$ & 11 & 3.03 & 3.42 \\
\hline 1 & 3 & 7 & 3.10 & 2.97 \\
\hline 0 & 0 & 12 & 2.41 & 2.51 \\
\hline 2 & -1 & 12 & 1.60 & 1.92 \\
\hline
\end{tabular}


Table 3. Final positional and thermal parameters (etc.).

a. Positional parameters.

$b$. Thermal parameters. ( $\beta_{13}$ and $\beta_{23}$ for oxygen were originally found to be relatively small and were therefore put equal to zero in later refinements).

c. R.m.s. amplitudes of vibration along principal axes.

E.s.d. in parentheses

$a$.

$\begin{array}{cccc} & x & y & z \\ \mathrm{~K} & 0.0 & 0.0 & 0.5 \\ \mathrm{~N} & (0) & (0) & (0) \\ \mathrm{O} & 0.0733 & \frac{1}{2} x & -0.010 \\ & 0.0728 & 0.2421 & -0.0266 \\ & (0.0044) & (0.0044) & (0.0029)\end{array}$

$b$.

$\begin{array}{ccccccc} & \beta_{11} & \beta_{22} & \beta_{33} & \beta_{12} & \beta_{13} & \beta_{23} \\ \mathrm{~K} & 0.1018 & \beta_{11} & 0.0144 & \beta_{11} & 0.0 \text { (ass.) } & 0.0 \text { (ass.) } \\ & (0.0024) & & (0.0004) & & & 0.0 \\ \mathrm{O} & 0.1315 & 0.1385 & 0.0241 & 0.1379 & 0.0 & 0.0 \\ & (0.0344) & (0.0180) & (0.0032) & (0.0468) & (0) & (0) \\ & B(\text { is })\left(\AA^{2}\right) & & & & & \\ \mathrm{N} & 11.0 & & & & & \end{array}$

$c$.

$\begin{array}{cclll} & \text { R.m.s. } & U(x) & U(y) & U(z) \\ & \text { Ampl. }(\AA) & (\AA) & (\AA) & (\AA) \\ & & & & \\ \mathrm{K} & 0.312 & 0.312 & 0.0 & 0.0 \\ & 0.312 & \frac{1}{2} u(y) & 0.352 & 0.0 \\ & 0.274 & 0.0 & 0.0 & 0.274 \\ & & & & \\ \mathrm{~N} & 0.374 & 0.374 & 0.0 & 0.0 \\ & 0.374 & \frac{1}{2} u(y) & 0.432 & 0.0 \\ & 0.374 & 0.0 & 0.0 & 0.374 \\ \mathrm{O} & & & & \\ & 0.352 & 0.361 & 0.0 & 0.0 \\ & 0.364 & 0.197 & 0.420 & 0.0 \\ & 0.355 & 0.0 & 0.0 & 0.355\end{array}$

The thermal parameters were treated as in the previous calculation, while the nitrogen atom was treated isotropically, as convergence could not be achieved with the use of anisotropic temperature factors when this atom was placed outside the origin. By this procedure, an $R$-factor of $5.32 \%$ was obtained. The corresponding observed and calculated structure factors are given in Table 2 . The parameter values listed in Table 3 differ only slightly from those obtained above. The corresponding intra- and intermolecular distances are contained in Table 4 . The intrinsic molecular data of the nitrite group are based on the results of the previous refinement. In the approximate evaluation of standard deviations in interatomic distances and angles, the estimated value of standard deviation of the $y$ parameter of the oxygen atom was equated to that of the $x$ parameter of this atom and of the nitrogen atom, 
Table 4. Intra- and inter-molecular distances based on data given in Table 3. E.s.d. in parentheses. (See Fig. 2b).

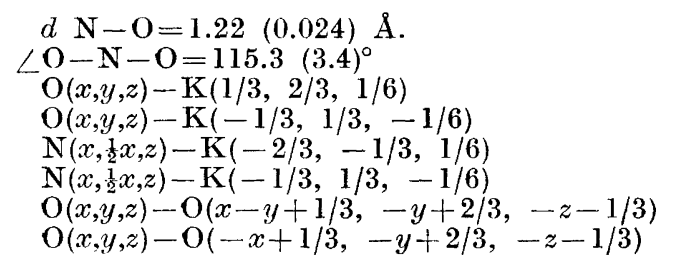

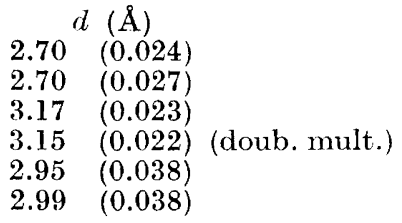

respectively, whereas the estimated error in the $z$ parameter of the oxygen atom was put equal to that of the $z$ parameter of the nitrogen atom.

The dimensions of the nitrite group are not significantly different from those obtained for sodium nitrite. ${ }^{10}$ Each oxygen atom is in close contact with two potassium ions. A similar $\mathrm{K}-\mathrm{O}$ distance has been observed in the trigonal modification of potassium nitrate. The $\mathrm{N}-\mathrm{K}$ distances are seen to be rather long. This is thought to be due largely to the excess positive charges on the atoms which were mentioned previously. The shortest statistical intermolecular $\mathrm{O}-\mathrm{O}$ distance is $2.95 \AA$. In the ordered structures of sodium nitrite and in nitrates the shortest $\mathrm{O}-\mathrm{O}$ separations are larger, about $3.3 \AA$ or more. However, since twice the van der Waals' radius $(2.8 \AA)$, is considerably less than $2.95 \AA$, the shortest $\mathrm{O}-\mathrm{O}$ distances may allow the corresponding relative orientations of the nitrite groups to become accessible to the system at equilibrium.

The magnitudes of the thermal parameters obtained for the nitrogen and oxygen atoms are less than the corresponding figures obtained in the L.S. refinement, in which the atoms were placed at the origin and in a mirror plane, respectively. This is thought to reflect an improvement in the present result compared with the original one. The thermal motion of the light atoms is more vigorous than that of the potassium ion, while the apparent vibration of the oxygen atom is found to be nearly isotropic. The motion of the potassium ion is rather more pronounced in directions perpendicular to the $z$ axis than parallel to it.

The instantaneous position of the nitrite group (etc.) considered above is actually an average (even if molecular vibration is ignored). At equilibrium, a system consisting of a central nitrite group and its six nearest potassium neighbours possesses mirror plane symmetry. The configuration is clearly sixfold degenerate.

Interaction with the rest of the lattice will in general break down the mirror plane symmetry. In principle, there is one distinct equilibrium orientation of the nitrite group for each of the many accessible configurations of the surroundings, although the various orientations of the nitrite group may in general differ only slightly from each other. It is the average which is observed.

Each nitrite group occupies, on the time average, six equally probable mean positions. This is accomplished by reorientation (rotation) of the nitrite groups. In accordance with the results outlined above, this motion may be expected to be hindered and, to some extent, coupled. This may alternatively 
be interpreted in terms of strongly directional attractive and repulsive forces. Because of the mutual repulsion between oxygen atoms, configurations involving longer intermolecular $\mathrm{O}-\mathrm{O}$ distances may be more probable than others (see, e.g., Fig. 2b) and give rise to a degree of short range order.

The heat of transition associated with the phase transformation at $-13^{\circ} \mathrm{C}$ has been reported to be $1200 \pm 200 \mathrm{cal} / \mathrm{mole}^{5}$ Relatively little attenuation of intensity of the $\mathrm{X}$-ray reflections for high values of $\theta$ is observed in the low temperature phase which probably shows no rotational disorder.

Although the value of the shortest intermolecular $\mathrm{O}-\mathrm{O}$ distance is only $2.95 \AA$, all statistically implied configurations will be considered to be accessible in calculating the configurational entropy. It is assumed further that the entropy may be separated into "configurational" and "thermal" parts. The number of distinct configurations of molecules in the lattice is $g^{N}$ where $N$ is the number of molecules considered, and $g$ (equal to 6 for the room temperature phase, and 1 for the low temperature phase, respectively) is the number of distinct positions of each nitrite group considered independently of its neighbours. The increment in configurational entropy between the low and room temperature phases is accordingly

$$
\Delta S_{\mathrm{c}}=k \ln 6^{N}=3.56 \mathrm{cal} / \mathrm{mole} \mathrm{deg} .
$$

compared with the experimental value of $4.62 \pm 0.77 \mathrm{cal} / \mathrm{mole} \mathrm{deg}$. This indicates that the entropy of transition is predominantly due to an increase in configurational entropy. The low temperature phase exhibits a molecular volume of $62.0 \AA^{3}$ at $-20^{\circ} \mathrm{C}$, while that obtained at room temperature is approximately $18.7 \%$ greater. Although the change at the transition temperature is a little smaller, it is still a relatively large quantity and indicates that the transformation is associated with an increase in potential energy accompanied by a considerable change in the vibrational state of the system, and possibly one which involves the onset of inversion of the nitrite group about the nitrogen atom.

\section{THE CUBIC STRUCTURE OF POTASSIUM NITRITE}

A continuous transformation from the trigonal phase at about $40^{\circ} \mathrm{C}$ results in a cubic phase with the unit cell dimension $a=6.66 \AA$ at $45^{\circ} \mathrm{C},{ }^{8}$ corresponding to four molecules per unit cell. In hexagonal indexing, the unit cell dimensions of the cubic phase become $a=4.71 \AA$ and $c=11.54 \AA$ compared with the room temperature values of $5.01 \AA$ and $10.15 \AA$, respectively. Thus the $a$ axis is seen to decrease and the $c$ axis to increase upon transformation to the cubic phase. The corresponding change in molecular volume from 73.85 to 73.60 $\AA^{3}$ is very small, however. In the cubic phase, the hexagonal $c$-axis becomes one of the four equivalent space diagonals of the unit cell. The trigonal structure corresponds to a face centred cubic structure of the sodium chloride type, thus if a nitrite group is statistically positioned about $\left(\frac{1}{2}, \frac{1}{2}, \frac{1}{2}\right)$, the six statistically nearest potassium neighbours will be situated at the centres of the six faces of the cubic unit cell, and thus form a regular octahedron. If the centre of symmetry and the mirror plane symmetry of the trigonal structure are retained in the continuous transformation, the point group symmetry of 
the cubic structure will be $m 3 m$. For a face centred structure we then obtain the space group $F m 3 m$. The following discussion is based on this space group.

However, a similar orientation of the nitrite group relative to the potassium ions to that described for the trigonal phase (involving only one close one-to-one $\mathrm{K}-\mathrm{O}$ contact in the cubic case) yields two equivalent $\mathrm{N}-\mathrm{K}$ distances of $2.89 \AA$, when an $\mathrm{O}-\mathrm{K}$ distance of $2.70 \AA$, and the same dimensions of the nitrite group as before are used. Compared with the corresponding $\mathrm{N}-\mathrm{K}$ distances obtained in the trigonal phase, this value seems too small to represent a relative equilibrium configuration of these atoms.

If the oxygen atoms are placed (in mirror planes) equidistant from two and two potassium ions, more satisfactory $\mathrm{N}-\mathrm{K}$ distances may be obtained. These oxygen atom positions can actually be exact only as an average, since the nitrite group as a whole is not in a special position in the unit cell, although the electrostatic attraction between the oxygen atom and its nearest potassium neighbours will tend to establish a symmetrical configuration of the oxygen atoms. As a result, the oxygen atoms may be observed to be markedly anisotropic. It appears that two non-equivalent orientations of the nitrite group may thus be obtained, in which the nitrogen atom is situated close to the origin, and six $\mathrm{N}-\mathrm{K}$ distances of approximately $a / 2=3.33 \AA$ are obtained. Each of these orientations gives rise to a total number of sixteen equivalent positions of the nitrite group. The positional parameters of Table 5 were obtained on the basis of an $\mathrm{O}-\mathrm{K}$ distance of $2.70 \AA$ and the nitrite group dimensions used previously. A model of the structure is shown in Fig. 3.

If we ignore the possibility that configurations implied statistically may actually be inaccessible for sterical reasons, the configurational entropy of the cubic phase, corresponding to an equal population of the two positional sets (relative to a "non positional disordered" structure) will be

$$
\Delta S_{\mathrm{c}}=R \ln 32=7.44 \mathrm{cal} / \mathrm{mole} \mathrm{deg} .
$$

In principle, the proposed model of the cubic structure seems to be the only one which is acceptable on the present basis, although neglected factors, such as intermolecular $\mathrm{O}-\mathrm{O}$ interactions, may modify the actual equilibrium positions of the molecules, as discussed for the trigonal phase.

Table 5. Atomic positions in the cubic unit cell, as deduced on the basis of results obtained for the trigonal phase. The $\mathrm{K}-\mathrm{O}$ contact distances assumed equal to $2.70 \AA$. The shortest $\mathrm{N}-\mathrm{K}$ distances are all nearly equal to $3.33 \AA(=a / 2)$.

a.

$\begin{array}{lrcc} & x & y & z \\ \mathrm{~K} & 0.500 & 0.0 & 0.0 \\ \mathrm{~N} & \sim 0.000 & \sim 0.000 & \sim 0.000 \\ \mathrm{O}_{1} & 0.120 & -0.120 & 0.076 \\ \mathrm{O}_{2} & -0.076 & 0.120 & \\ & & & 0.120 \\ \mathrm{~K} & 0.500 & 0.0 & 0.0 \\ \mathrm{~N} & \sim 0.000 & \sim 0.000 & \sim 0.000 \\ \mathrm{O}_{1} & 0.120 & -0.120 & -0.076 \\ \mathrm{O}_{2} & 0.076 & 0.120 & 0.120\end{array}$

Acta Chem. Scand. 23 (1969) No. 1 
Fig. 3. Model of the cubic structure of potassium nitrite. The nitrite group is shown in two non-equivalent positions with the nitrogen atom placed close to the centre of a regular octahedron and the six potassium ions at the corners. Cubic axes.

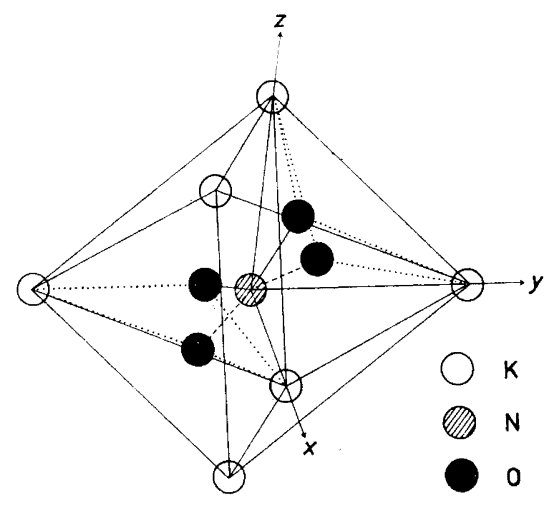

THE PHASE DIAGRAM OF $\mathrm{KNO}_{2}, \mathrm{KNO}_{3}$ AND $\mathrm{H}_{2} \mathrm{O}$ AT ROOM TEMPERA'TURE

According to Ray, commercially available potassium nitrite contains from 3 to $5 \%$ by weight of $\mathrm{KNO}_{3} \cdot{ }^{4}$ The present sample contained about $3 \% \mathrm{KNO}_{3}$. The solubility of $\mathrm{KNO}_{3}$ at $20^{\circ} \mathrm{C}$ is estimated to be approximately $15 \%$.

Above $128^{\circ} \mathrm{C}$ potassium nitrate acquires a statistical trigonal structure ${ }^{16}$ which resembles the present one. The following unit cell dimensions were obtained from the reported high temperature data ${ }^{17}$ by extrapolating to room temperature.

$$
a=b=5.428 \AA ; \quad c=9.79 \AA
$$

The values correspond to a molecular volume of $79.07 \AA^{3}$ (which is about $1 \%$ less than the value for the room temperature phase. ${ }^{17}$ For comparison, the molecular volume of potassium nitrite is $73.72 \AA$, which is $6.8 \%$ less than that quoted for $\mathrm{KNO}_{3}$. As both the statistical structures and effective molecular volumes are observed to be similar in the two cases, the energy needed to form a solid solution is relatively small and may be compensated for by the corresponding increase in (configurational) entropy. Potassium nitrite is, on the other hand, practically insoluble in the orthorhombic room temperature phase of potassium nitrate, ${ }^{3}$ the (ordered) structure of which is different. ${ }^{18}$

Fig. 4 shows the phase diagram of the ternary system $\mathrm{KNO}_{2}, \mathrm{KNO}_{3}$, and $\mathrm{H}_{2} \mathrm{O}$ at $25^{\circ} \mathrm{C}$ and atmospheric pressure. ${ }^{3}$ The equilibrium between the solid solution of $\mathrm{KNO}_{3}$ in $\mathrm{KNO}_{2}$ (s. sol.) and the aqueous solution (sol.) may be expressed as

and

$$
\begin{aligned}
& K_{\mathrm{I}}=a_{\mathrm{KNO}_{2} \text { (sol.) }} / a_{\mathrm{KNO}_{8} \text { (s. sol.) }} \\
& K_{\mathrm{II}}=a_{\left.\mathrm{KNO}_{3} \text { (sol.) }\right)} / a_{\mathrm{KNO}_{3} \text { (s. sol.) }}
\end{aligned}
$$

where $K_{\mathrm{I}}$ and $K_{\mathrm{II}}$ are equilibrium constants and $a$ stands for activity. The ratios of the mole fractions of aqueous $\mathrm{KNO}_{2}$ and solid $\mathrm{KNO}_{2}$ are 0.391 and 0.404 , corresponding to the positions $\mathrm{A}$ and $\mathrm{B}$ on the phase diagram, respectively. The constancy of this ratio indicates that the ratio of the activity

Acta Chem. Scand. 23 (1969) No. 1 


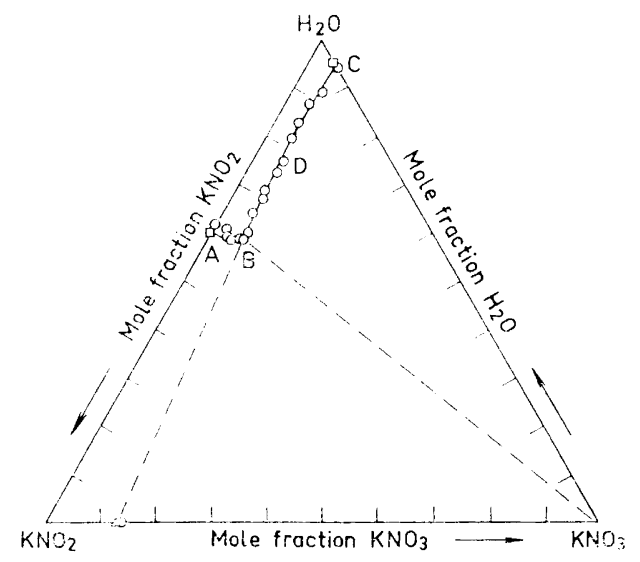

Fig. 4. The phase diagram of $\mathrm{KNO}_{2}, \mathrm{KNO}_{3}$ and $\mathrm{H}_{2} \mathrm{O}$ at room temperature $\left(25^{\circ} \mathrm{C}\right)$. O: Ref. 3.

$\square$ : Handbook of Chemistry.

$\triangle$ : Present work $\left(20^{\circ} \mathrm{C}\right)$.

coefficients is also nearly constant in the region. Hence the approximate composition of the solid solution may be found once the mole fraction of $\mathrm{KNO}_{2}$ in the corresponding liquid phase is known.

In view of the marked effect of ionic strength upon the activity coefficient, it is surprising to find the mole-fraction of $\mathrm{KNO}_{3}$ approximately constant in the region $\mathrm{B}$ to $\mathrm{C}$, which represents the composition of the aqueous solution in equilibrium with pure solid $\mathrm{KNO}_{3}$.

According to Bureau, ${ }^{3}$ a rhombohedral $\beta$-form of $\mathrm{KNO}_{3}$ crystallizes from an aqueous solution in the region from $B$ to $D$, which is not a well defined point on the curve. We have found that crystals of $\mathrm{KNO}_{3}$ grown from a solution close to that of the point $B$ appear to be orthorhombic and of practically the same unit cell dimensions as those reported for the $\alpha$-form grown from an aqueous solution of $\mathrm{KNO}_{3}$ which was free from $\mathrm{KNO}_{2} \cdot{ }^{17}$ The results obtained for the $\alpha$ - and $\beta$-forms at room temperature are, respectively,

$$
\begin{array}{lll}
a=5.4175(0.0010) \AA & b=9.1709(0.0007) \AA \\
c=6.4255(0.0008) \AA & V=319.240 \AA 17 \\
a=5.398(0.001) \AA & b=9.122(0.002) \AA \\
c=6.452(0.001) \AA & V=317.70 \AA
\end{array}
$$

The difference between corresponding figures is seen to be about $1 \%$ or less. The unit cell volume is seen to be slightly less in the present case which may indicate the presence of traces of $\mathrm{KNO}_{2}$ in this sample. The existence of a phase transformation in solid $\mathrm{KNO}_{3}$ corresponding to the point $\mathrm{D},{ }^{3}$ does not therefore appear to be likely.

The full matrix least-squares computer programme used is a somewhat revised version of that written by Gantzel, Sparks and Trueblood (IUCr World List No.384). The Fourier programme was written by Gantzel and Hope (Department of Chemistry, University of California, Los Angeles, Calif., U.S.A.).

We wish to thank cand. real. Rolf Møllerud for the Guinier photographs used in the determination of the unit cell dimensions. 


\section{REFERENCES}

1. Chang, Shih-Chi, Diss. Abstr. 24 (1963) 1668.

2. Tanisaki, S. and Ishimatsu, T. J. Phys. Soc. Japan 20 (1965) 1277.

3. Bureau, J. Ann. Chim. 8 (1937) 5.

4. Ray, J. D. J. Inorg. Nucl. Chem. 15 (1960) 290.

5. Ray, J. D. and Ogg, R. A. J. Phys. Chem. 60 (1956) 1599.

6. Parry, G. S., Schuyff, A. and Ubbelohde, A. R. Proc. Roy. Soc. 285 (1965) 360.

7. Ziegler, G. E. Z. Krist. 94 (1936) 491.

8. Parry, G. S. and Schuyff, A. Acta Cryst. 21 (1966) 303.

9. Abeledo, C. A. and Kolthoff, I. M. J. Am. Chem. Soc. 53 (1931) 2893.

10. Kay, M. I., Frazer, B. C. and Ueda, R. Acta Cryst. 15 (1962) 506.

11. Hanson, H. P., Herman, F., Lea, J. D. and Skillman, S. Acta Cryst. 17 (1964) 1040.

12. Weiss, A. and Hattenbach, K. Z. physik. Chem. (Frankfurt) 29 (1961) 293.

13. Sawada, S., Nomura, S., Fuhi, S. and Yoshida, I. Phys. Rev. Letters 1 (1958) 230.

14. Cruickshank, D. W. J. Acta Cryst. 14 (1961) 896.

15. Srinivasan, R. Acta Cryst. 14 (1961) 1163.

16. Tahvonen, P. E. Ann. Acad. Sci. Fennicae Ser. A 1947 No. 44.

17. Fischmeister, H. F. J. Inorg. Nucl. Chem. 2 (1956) 182.

18. Edwards, D. A. Z. Krist. 80 (1931) 154.

Received June 12, 1968. 